\title{
\begin{tabular}{l|l} 
Mitraries & DSpace@MIT
\end{tabular}
}

\author{
MIT Open Access Articles
}

\section{Analog and Digital Deformable Diffractive Optics Actuated by Inter-Nanomagnet Forces}

The MIT Faculty has made this article openly available. Please share how this access benefits you. Your story matters.

Citation: Deterre, Martin et al. “Analog and Digital Deformable Diffractive Optics Actuated by Inter-Nanomagnet Forces." IEEE Journal of Quantum Electronics 46.9 (2010) : 1275-1287. (C) Copyright 2010 IEEE

As Published: http://dx.doi.org/10.1109/jqe.2010.2046721

Publisher: Institute of Electrical and Electronics Engineers

Persistent URL: http://hdl.handle.net/1721.1/67461

Version: Final published version: final published article, as it appeared in a journal, conference proceedings, or other formally published context

Terms of Use: Article is made available in accordance with the publisher's policy and may be subject to US copyright law. Please refer to the publisher's site for terms of use. 


\title{
Analog and Digital Deformable Diffractive Optics Actuated by Inter-Nanomagnet Forces
}

\author{
Martin Deterre, Anthony J. Nichol, Se Baek Oh, and George Barbastathis
}

\begin{abstract}
In this paper, we present a novel actuation technique of diffractive optics based on magnetic interaction forces, permitting a fine resolution tuning as well as a tuning in discrete steps. This actuation combines advantages of "analog" and "digital" tuning in conventional diffractive optics. The application of a magnetic field perpendicularly to the beams of a freestanding grating coated with magnetic material generates intermagnet forces causing these beams to deform slightly or to clump together. A theoretical analysis of the forces has been carried out, followed by actuation simulations in both linear and nonlinear regimes for different types of gratings, showing examples of exponential and parabolic chirp profiles in addition to beam clumping. In experiment, the diffraction patterns of distinct fabricated gratings have shown both a continuous shift and smear as well as a discrete change in the diffracted angles proving the concept of analog and digital tuning.
\end{abstract}

Index Terms-Analog tuning, diffraction gratings, digital tuning, magnetic actuation, microelectromechanical systems (MEMS), nanomagnets.

\section{INTRODUCTION}

$\mathbf{T}$ UNABLE diffractive elements are interesting for a broad range of optical microelectromechanical systems. Common applications are found in visual displays [1], [2], scanners, miniaturized spectrometers [3], external cavity tunable lasers [4], switches, and wavelength tuning elements in dense wavelength-division multiplexing [5], and active compensation devices for thermal disturbances in optical instruments. Current actuation techniques for diffraction gratings can be classified as either "digital" or "analog" (Fig. 1). Digital tuning is based on the binary actuation of the grating beams, as applied by the grating light valve [1], [2], [6], where the out-ofplane movement of individual grating beams can be controlled through electro-static forces. This technique can achieve highfrequency tuning but its actuation range is limited to discrete steps corresponding to the multiples of the beam width. On the other hand, analog actuation permits a much finer resolution reaching a fraction of a percent of the beam width

Manuscript received December 15, 2009; revised February 9, 2010; accepted March 22, 2010. Date of current version July 23, 2010. This work was supported in part by the Air Force Office of Scientific Research, Multidisciplinary Research Initiative: Membrane Technology and in part by 3-D Photonic-Crystal Devices, under Contract FA 9550-08-1-0379. This paper was recommended by Associate Editor H. P. Herzig.

The authors are with the Department of Mechanical Engineering, Massachusetts Institute of Technology, Cambridge, MA 02139 USA (e-mail: deterre@mit.edu; anichol@mit.edu; sboh@mit.edu; gbarb@mit.edu).

Color versions of one or more of the figures in this paper are available online at http://ieeexplore.ieee.org.

Digital Object Identifier 10.1109/JQE.2010.2046721
[3], [6], [7]. Analog tuning is typically achieved via one of the following principles: electrostatic actuation of a structure held by flexures [3], [6], [8]-[10], piezo-electric [7], [11] or electrostatic stretching of a membrane [12], deformations of thermal actuators [13]-[15], electrostatic actuation for variable blaze angles [16], [17] or thermal/magnetic actuation of fiber Braggs gratings [18]. However, these tuning techniques trade the large actuation range of the digital actuation for analog precision.

Here, we present a method aiming to combine the advantages of both techniques by coating diffraction gratings with nanomagnets (Fig. 2). Previous work showed that nanomagnets can fold, actuate, and align microelectromechanical systems (MEMS) components including optical features [19], [20]. External magnetic fields can adjust inter-nanomagnet forces to provide either small forces or strong pull-in forces. This method enables several actuation regimes where a single diffraction grating can be tuned in either digital or analog fashion, effectively expanding its actuation range.

The main advantage of the magnetic force over the electrostatic is that pull-in is allowed as there are no shortcircuits possible. Furthermore, a bundle of clumped grating beams can still be actuated the same way single beams are, enabling supplementary fine-tuning or pull-in effects. Certain designs of nanomagnet patterns also allow repulsive scheme to increase further the tuning range. Since the magnetic coating is needed only on the grating beams, the actuation features are embedded in the device structure and additional space for flexures or electrostatic combs is not required. This enables actuation of other micro or nano-structured devices where space constraints are critical. Moreover, magnetic actuation can exhibit nontrivial deformed profiles such as exponential or parabolic chirp, expanding the spectrum of possible applications including tunable metamaterials. Fig. 3 shows a schematic example of an aperiodic tunable device from an initially periodic structure. Chirping this structure would produce an effective index profile emulating a tunable gradientindex (GRIN) structure, such as a cloak [21]-[23] or lens [24], [25].

Nevertheless, an auxiliary system is required to produce the magnetic field, and a precise control over the magnetic force remains challenging due to the shape anisotropy of the nanomagnets magnetization.

In this paper, we introduce a theoretical analysis of the grating deformations under magnetic actuation, numerical 

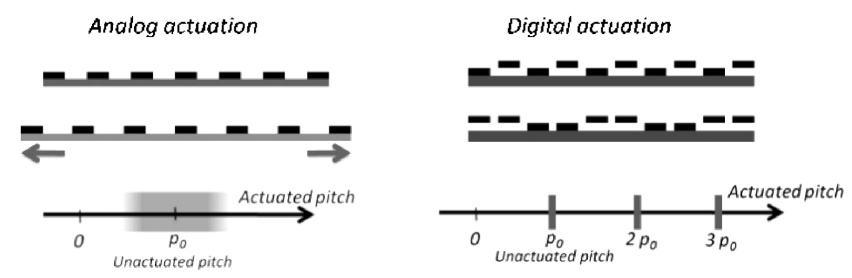

Fig. 1. Actuation concept and range for analog and digital tunable gratings.

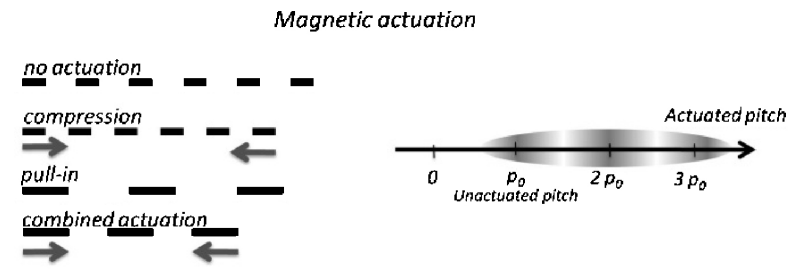

Fig. 2. Actuation concept and range for magnetically actuated gratings.

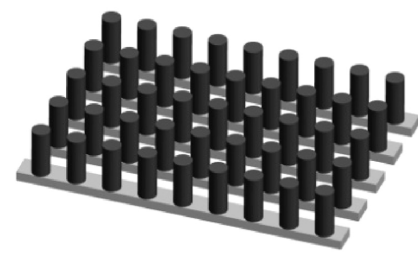

(a)

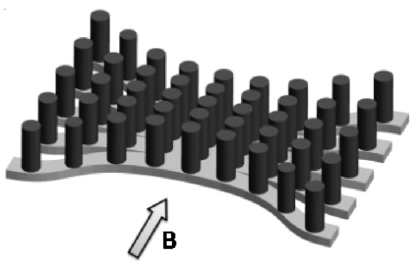

(b)
Fig. 3. Schematic of a periodic photonic structure on a (a) freestanding grating (b) tuned to an aperiodic structure under magnetic actuation.

simulations showing the shape of gratings having different parameters and designs, and experiments proving the concept of this magnetically actuated grating for both analog and digital tunings.

\section{CONCEPT}

A grating is made of freestanding silicon nitride beams covered by a magnetic layer. As depicted in Fig. 4, this layer can be magnetized by an external magnetic field. When the direction of magnetization is in plane perpendicularly to the grating beams, opposite magnetic poles are created face to face across neighboring beams and become subjected to an attractive force. This interaction causes the beams to bend until equilibrium with the elastic resistance is reached. If the attraction is strong enough, neighboring beams clump together by pull-in effect.

Characterization of these deformations can be achieved by illuminating the grating at normal incidence and studying the changes in the diffraction pattern. In the case of small deformations, the diffraction orders shift and smear slightly because of the bent shape of the grating beams. In the case of beams clumping by pull-in, the diffraction orders jump in a discrete fashion to a fraction of the original angle.

To obtain interesting actuation shapes, different geometries of gratings are studied. A general representation of the grating and the notation used in the subsequent sections are described in Fig. 5. (a)
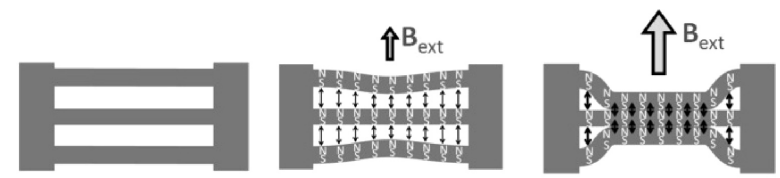

(b)
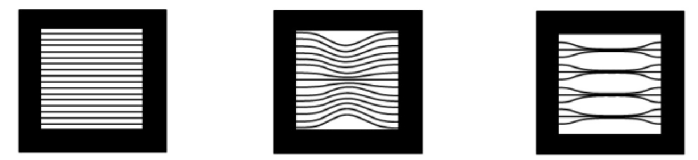

(c)
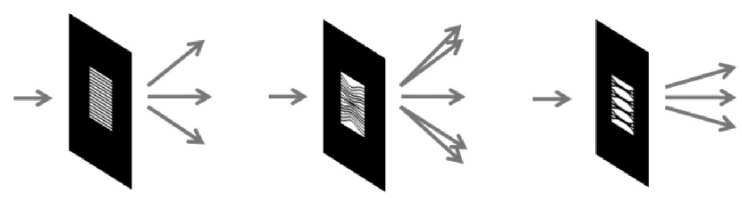

Fig. 4. Schematic principle of the magnetic actuation. (a) Top view of three grating beams attracted together when subjected to a perpendicular magnetic field. (b) Top view of a grating which slightly deforms for low magnetic field and whose beams can clump together for high magnetic field. (c) Diffraction of an incoming monochromatic light beam.

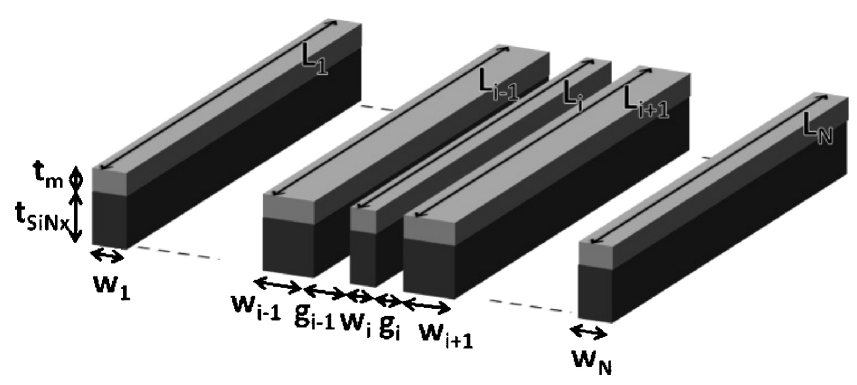

Fig. 5. Grating parameters and notation.

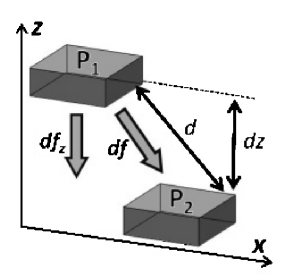

(a)

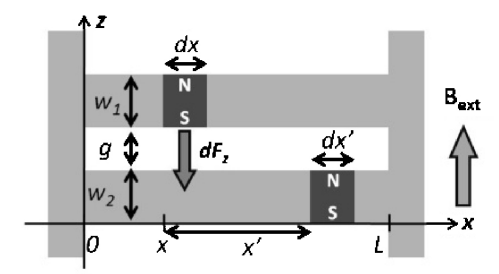

(b)
Fig. 6. (a) Schematic and notation of the force between two theoretical magnetic monopoles. (b) Schematic top view and notation of two neighboring beams split into elementary magnetic dipoles.

\section{ANALYSIS}

The shape of the actuated grating beams is the result of the equilibrium between the magnetic forces, the bending resistances, and the contact forces if beams are touching.

\section{A. Magnetic Force}

Let's consider two neighboring beams with length $L$, apart from a distance $g$ and coated with a magnetic layer of thickness $t_{m}$. To model the interaction force, we split them into small elements of width $d x$ as shown in Fig. 6(b). These elements can be approximated as magnetic dipoles with pole strength $P= \pm \mu_{0}^{-1} M A$ [19], where $\mu_{0}$ is the air permeability (assumed equal to vacuum permeability), $M$ is the material magnetization in Tesla, and $A$ is the cross-section area perpendicular 
to the magnetization. Consider two magnetic poles $P_{1}, P_{2}$ at distance $d$ as illustrated in Fig. 6(a). The force between these poles can be written as $d f=\mu_{0} P_{1} P_{2} /\left(4 \pi d^{2}\right)$. Projecting along the vertical axis gives $d f_{z}=\mu_{0} P_{1} P_{2} \cdot d z /\left(4 \pi d^{3}\right)$, where $d z$ is the vertical gap between the poles. Taking into account the four interactions of the four poles, the total magnetic force between the two elements is

$$
\begin{gathered}
d F_{z}\left(x, x^{\prime}\right)=\frac{M^{2} \cdot t_{m}^{2} \cdot d x \cdot d x^{\prime}}{4 \pi \mu_{0}}\left(\frac{g}{\left(x^{\prime 2}+g^{2}\right)^{\frac{3}{2}}}-\frac{g+w_{1}}{\left(x^{\prime 2}+\left(g+w_{1}\right)^{2}\right)^{\frac{3}{2}}}\right. \\
\left.-\frac{g+w_{2}}{\left(x^{\prime 2}+\left(g+w_{2}\right)^{2}\right)^{\frac{3}{2}}}+\frac{g+w_{1}+w_{2}}{\left(x^{\prime 2}+\left(g+w_{1}+w_{2}\right)^{2}\right)^{\frac{3}{2}}}\right)
\end{gathered}
$$

The force per unit length exerted by the whole lower beam on the upper beam element at position $x$ is then

$$
\begin{aligned}
F_{\operatorname{lin}}(x) & \equiv \frac{d F_{z}(x)}{d x}=\int_{x^{\prime}=-x}^{x^{\prime}=L-x} \frac{d F_{z}\left(x, x^{\prime}\right)}{d x} \\
& =\int_{-x}^{L-x} \frac{M^{2} \cdot t_{m}^{2} \cdot d x^{\prime}}{4 \pi \mu_{0}}\left(\frac{g}{\left(x^{\prime 2}+g^{2}\right)^{\frac{3}{2}}}-\frac{g+w_{1}}{\left(x^{\prime 2}+\left(g+w_{1}\right)^{2}\right)^{\frac{3}{2}}}\right. \\
& \left.-\frac{g+w_{2}}{\left(x^{\prime 2}+\left(g+w_{2}\right)^{2}\right)^{\frac{3}{2}}}+\frac{g+w_{1}+w_{2}}{\left(x^{\prime 2}+\left(g+w_{1}+w_{2}\right)^{2}\right)^{\frac{3}{2}}}\right) .
\end{aligned}
$$

This can be integrated analytically and yields a long expression, which away from the edges (i.e., $x$ and $L-x \gg$ $\left.g, w_{1}, w_{2}\right)$ is independent of $x$ and equals to

$$
F_{\text {lin }}=\frac{M^{2} \cdot t_{m}^{2}}{2 \pi \mu_{0}}\left(\frac{1}{g}-\frac{1}{g+w_{1}}-\frac{1}{g+w_{2}}+\frac{1}{g+w_{1}+w_{2}}\right) .
$$

As the aspect ratio of the beams $\mathrm{L} / \mathrm{w}$ is usually very large (typically 500:1), the side effects are considered negligible and we subsequently assume the latter expression to be valid along the entire beam.

This analysis remains approximately valid even if the beams are slightly bent, i.e., the deflection $\delta$ is small compared to the gap $g$. If $w_{1}=w_{2}=w$, this condition can be refined to

$$
\delta \ll g\left(1+\frac{g}{w}\right)\left(1+\frac{g}{2 w}\right) .
$$

\section{B. Magnetization Analysis}

Since the aspect ratio of the beams is very high, the magnetic coating has an inclination to be magnetized along the beams. Therefore, a relatively strong magnetic field is required to force the magnetization to be in the perpendicular direction. Fig. 7 describes an object oriented micromagnetic framework (OOMMF) simulation of the magnetization of three beams coated with $20 \mathrm{~nm}$ of cobalt. It shows that a field of approximately $0.1 \mathrm{~T}$ is sufficient to set the magnetization orientation perpendicular to the grating beams.

This shape factor limits our tuning ability over the magnetic force as the relationship between the external field strength and the vertical component of the magnetization is mostly
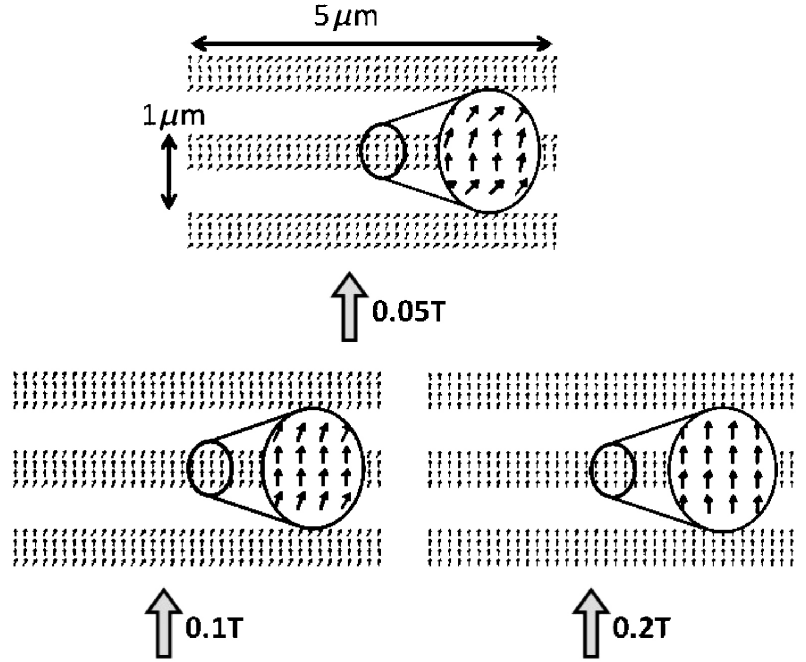

Fig. 7. OOMMF simulation of the magnetization of a grating beams coating made of $20 \mathrm{~nm}$ of cobalt.

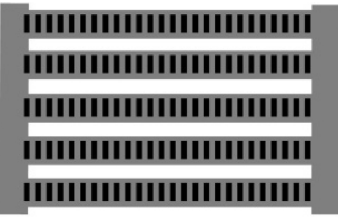

- Silicon nitride

(a)

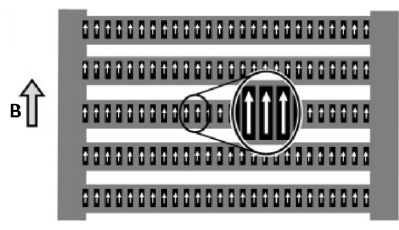

iी Internal magnetization

(b)
Fig. 8. Schematics of patterned nanomagnets rows. (a) Atop the grating. (b) With their internal magnetization.

nonlinear. To increase controllability, one might shape the magnetic material into rows of thin vertical nanomagnets as illustrated in Fig. 8 instead of a uniform coating. Having turned the aspect ratio factor into our advantage, now even a small magnetic field is able to magnetize the nanomagnets perpendicularly to the beams. This substantially simplifies the relationship between the strength of the applied magnetic field and the perpendicular magnetic force, enabling a much more significant tuning ability.

In the subsequent simulations and experiments of this article, only uniform magnetic coatings are involved and it is assumed that the external field is strong enough to magnetize the magnetic layer to saturation and fully orient it perpendicularly.

\section{Beam Distortion Analysis}

The magnetic forces are applied to the beam coatings on top of the beams. Hence, the action on each beam is two-fold: a torsion moment and an in-plane force, as depicted in Fig. 9.

In this model, the magnetic force is represented by a load per unit length $F_{l}$ at a height $h$ from the central axis of the beam of length $L$ [Fig. 9(a)]. This load is decoupled into a moment $M_{l}$ and a force $F_{l}$, both per unit length, applied at the central axis [Fig. 9(b)]. We have

$$
M_{l}=F_{l} \cdot h=\frac{F_{l} \cdot t_{s}}{2} .
$$




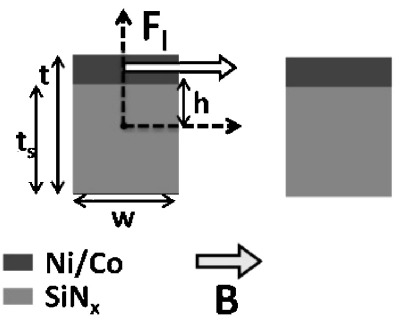

(a)

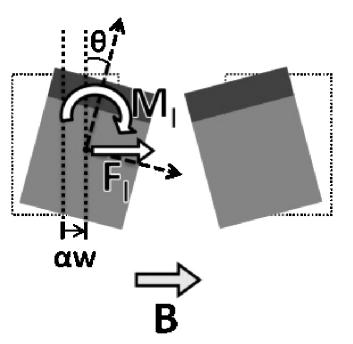

(b)
Fig. 9. Cross-section schematics of two attracting neighboring beams. (a) Undistorted case. (b) Distorted case showing translation and torsion.

We know from structural mechanics [26] that the twist in a beam $d \theta / d x$ is governed by the following equation:

$$
M=G j \frac{d \theta}{d x}
$$

where $M=L \cdot M_{l}$ represents the total moment on the beam, $G$ is the beam shear modulus, and $J$ is the area moment of torsion inertia. In our case (rectangular cross-section) we have $J=$ $\beta t w^{3}$, where $\beta$ is a coefficient being in the order of 0.15 when $t$ and $w$ are similar. Also, $G$ can be expressed in terms of the Young's modulus $E$ and Poisson ratio v: $G=E /(2(1+v))$. We obtain from the previous equations that the maximum torsion angle $\theta$ at mid-length $L / 2$ is

$$
\theta=\frac{F_{l} t_{s} L^{2}(1+v)}{0.3 E t w^{3}} .
$$

Beam bending theory [27] is invoked to determine the inplane displacement $\alpha w$. At mid-point of the fixed-fixed beam, the deflection is equal to

$$
a w=\frac{L^{4}}{384 E I} F_{l}
$$

where $I$ is the area moment of inertia, in our case equal to $t w^{3} / 12$. It is assumed here that the Young's moduli of the nitride and of the magnetic material are similar, which is true for nickel and cobalt.

As $\alpha$ is a dimensionless variable measuring the significance of the deflection, the relative importance of the beam torsion over the beam deflection is determined by calculating the ratio of $\theta$ over $\alpha$

$$
\frac{\theta}{\alpha}=\frac{32 t_{s} w(1+v)}{0.3 L^{2}} .
$$

Because in our grating geometry, the beam cross-section dimensions are about five hundred times smaller than the beam length, the ratio $\theta / \alpha$ is estimated to be less than $10^{-3}$. Hence, we conclude that the torsion effect is negligible compared to the deflection effect. In the following sections, the movement of the beam will be considered as fully in plane, as if the magnetic force was applied at the central axis of the beam.

\section{First Order Analysis}

Two different equilibria come into play depending on whether the beams bundle or stay disconnected. To arbitrate between the two regimes, we first consider only two neighboring beams. Assuming that these beams are similar, they will deflect by the same amount in opposite directions. Hence, the

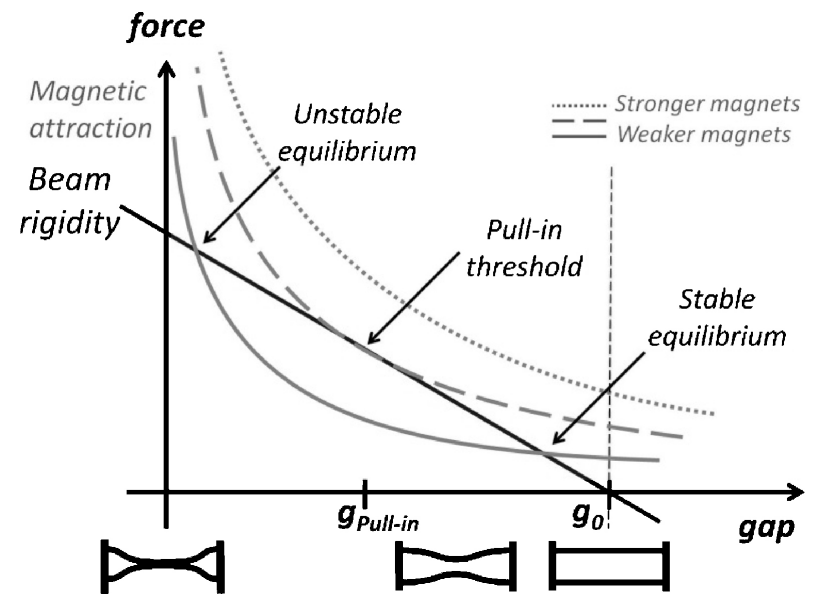

Fig. 10. First order analysis-balance of forces.

gap between the beams $g$ is expressed as $g_{0}-2 \delta$, where $g_{0}$ is the gap at rest and $\delta$ is the deflection of the beams. Assuming as a first order approximation that the load is constant along the beam, the beam under consideration acts as a spring whose stiffness at midpoint is

$$
k_{j} \equiv \frac{32 \cdot E \cdot t_{\text {total }} \cdot w_{j}^{3}}{L^{4}} \quad j=1,2 .
$$

Using a sign convention that assigns a positive sign for forces that increase the gap $g$, the net force on beam $j$ can then be expressed as

$$
\begin{aligned}
F_{\text {net }}= & \frac{-M^{2} \cdot t_{m}{ }^{2}}{2 \pi \mu_{0}}\left(\frac{1}{g}-\frac{1}{g+w_{1}}-\frac{1}{g+w_{2}}+\frac{1}{g+w_{1}+w_{2}}\right) \\
& +k_{j}\left(g_{0}-g\right) / 2 .
\end{aligned}
$$

Equilibrium is found when $F_{\text {net }}$ is zero, which occurs for two values of positive gaps. The equilibria stability is determined by the sign of the following expression:

$$
\begin{gathered}
\frac{\partial F_{\text {net }}}{\partial g}=\frac{M^{2} t_{m}^{2}}{2 \pi \mu_{0}}\left(\frac{1}{g^{2}}-\frac{1}{\left(g+w_{1}\right)^{2}}-\frac{1}{\left(g+w_{2}\right)^{2}}+\frac{1}{\left(g+w_{1}+w_{2}\right)^{2}}\right) \\
-\frac{k_{j}}{2} .
\end{gathered}
$$

For weak magnets $\left(M \cdot t_{m}\right.$ small), the latter expression is negative resulting in a stable equilibrium as illustrated in Fig. 10. Clearly, as the magnetic strength increases, there will be a gap for which (8) becomes always positive, meaning that the stability of the equilibrium is lost (pull-in effect). This is similar to the electrostatic pull-in effect, even though the electrostatic force has a simpler expression and varies as $1 / g^{2}$ [28].

The threshold gap $g_{P I}$ for which pull-in occurs can be found by setting (7) and (8) both equal to zero, yielding

$$
\begin{gathered}
\frac{1}{g_{P I}}-\frac{1}{g_{P I}+w_{1}}-\frac{1}{g_{P I}+w_{2}}+\frac{1}{g_{P I}+w_{1}+w_{2}} \\
=\left(\frac{1}{g_{P I}^{2}}-\frac{1}{\left(g_{P I}+w_{1}\right)^{2}}-\frac{1}{\left(g_{P I}+w_{2}\right)^{2}}\right. \\
\left.+\frac{1}{\left(g_{P I}+w_{1}+w_{2}\right)^{2}}\right)\left(g_{0}-g_{P I}\right) .
\end{gathered}
$$


Unfortunately, this equation cannot be solved symbolically. However, for our application where $w_{1}, w_{2}$, and $g_{0}$ have similar values (typically $500 \mathrm{~nm}), g_{P I}$ is numerically equal to about $g_{0} / 3$. Like in electrostatic pull-in, where $g_{P I}$ is equal to $2 g_{0} / 3$, it is interesting to see that $g_{P I}$ depends only on the geometry of the beams $\left(w_{1}, w_{2}\right.$, and $\left.g_{0}\right)$ and neither on the rigidity of the beam nor on the strength of the magnets. However, one has to be cautious with the value of $g_{P I}$, as for example at $g_{0} / 3$ the deflection is significant and some assumptions such as the uniformity of the magnetic load start to break.

Once the pull-in gap has been determined, the sign of (8) at $g_{P I}$ determines if we are in the slightly deformed case (presence of equilibrium) or in the clumped case (beam pull-in). By characterizing the magnetic layer strength as the product of magnetization and thickness $M \cdot t_{m} \equiv S$, the critical magnetization strength between the two cases for the $j$ th beam can be written as

$$
S_{j, \text { critical }}=\sqrt{\frac{\pi \mu_{0} 64 \cdot E \cdot t_{\text {total }} \cdot w_{j}^{3}}{L^{4}\left(\frac{1}{g_{P I}{ }^{2}}-\frac{1}{\left(g_{P I}+w_{1}\right)^{2}}-\frac{1}{\left(g_{P I}+w_{2}\right)^{2}}+\frac{1}{\left(g_{P I}+w_{1}+w_{2}\right)^{2}}\right)}} .
$$

Hence, if $S<S_{\text {critical }}$ the beams are in the small deformation regime, and if $S>S_{\text {critical }}$ they are in the so-called clumping regime.

\section{E. Small Deformation Regime}

As the gap between two beams depends on their relative deflections, the bending of one beam will affect the magnetic load and the bending of its neighbors. Therefore, determining the deformed grating profile requires the solution of a coupled system. Additionally, knowing the exact shape of the beams might be essential for applications such as diffractive optical elements or tunable photonic structures. For that reason the deflection at every point along the beams has to be determined. Again, we invoke beam bending theory stipulating that for a beam subjected to a deflection dependent per-unit length load $P_{l}$, the deflection $\delta$ at position $x$ follows the beam equation [27]:

$$
\frac{d^{4} \delta}{d x^{4}}(x)=\frac{12 \cdot P_{1}(\delta(x))}{E \cdot t_{\text {total }} \cdot w^{3}}
$$

The dependence of $P_{l}$ on $\delta(x)$ is being due to the dependence of the magnetic force on the interline gap.

For a weak magnetic layer, the next approximation is to linearize the load with respect to $\delta(x)$ around the unactuated position. For the typical geometries used in our experiments, (2) shows that magnetic strengths leading to deformation not exceeding $20 \%$ of the nominal (unactuated) gap, the error in force value due to linearization of (2) does not exceed $10 \%$.

The linearized magnetic force can be written as

$F_{\text {lin }}\left(g_{0}+d g, w_{1}, w_{2}\right)=\alpha\left(g_{0}, w_{1}, w_{2}\right)-d g \cdot \beta\left(g_{0}, w_{1}, w_{2}\right)$.

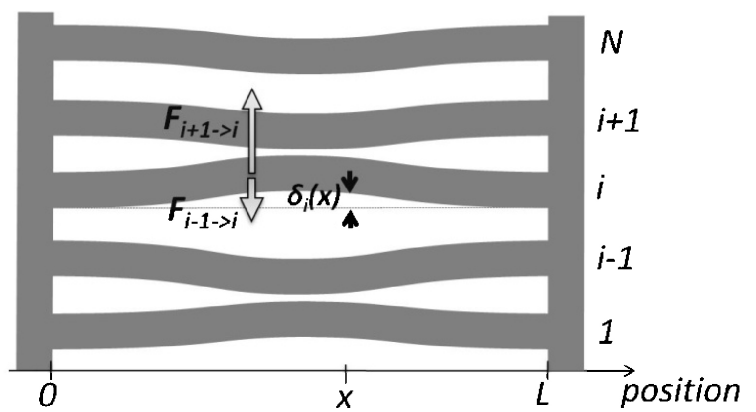

Fig. 11. Schematics of the magnetic forces on beam $i$.

Here $g_{0}$ is the nominal gap, $d g$ is the gap variation and the functions $\alpha, \beta$ are defined as follows:

$$
\begin{gathered}
\alpha\left(g_{0}, w_{1}, w_{2}\right) \\
\equiv \frac{M^{2} t_{m}^{2}}{2 \pi \mu_{0}}\left(\frac{1}{g_{0}}-\frac{1}{g_{0}+w_{1}^{2}}-\frac{1}{g_{0}+w_{2}}+\frac{1}{\left(g_{0}+w_{1}+w_{2}\right.}\right) \\
\beta\left(g_{0}, w_{1}, w_{2}\right) \\
\equiv \frac{M^{2} t_{m}^{2}}{2 \pi \mu_{0}}\left(\frac{1}{g_{0}^{2}}-\frac{1}{\left(g_{0}+w_{1}\right)^{2}}-\frac{1}{\left(g_{0}+w_{2}\right)^{2}}\right. \\
\left.+\frac{1}{\left(g_{0}+w_{1}+w_{2}\right)^{2}}\right) .
\end{gathered}
$$

In theory, two different beams are interacting even if they are not next to each other. However, the strength of the force between two non-adjacent beams is less than $10 \%$ of the strength of the force between adjacent beams. Hence in this analysis the linear load on the beam $i$ at position $x$ is limited to the contribution of the two adjacent beams $i-1$ and $i+1$ (Fig. 11).

From (11) and (12), we have for $2 \leq i \leq N-1$

$$
\begin{aligned}
& \frac{d^{4} \delta_{i}}{d x^{4}}(x)=\frac{12}{E \cdot t_{\text {total }} \cdot w_{i}{ }^{3}}\left(F_{i+1 \rightarrow i}\left(g_{i+1}+\delta_{i+1}(x)-\delta_{i}(x), w_{i}, w_{i+1}\right)\right. \\
& \left.\quad+F_{i-1 \rightarrow i}\left(g_{i}+\delta_{i}(x)-\delta_{i-1}(x), w_{i-1}, w_{i}\right)\right) \\
& \quad \Leftrightarrow \frac{d^{4} \delta_{i}}{d x^{4}}(x)=\frac{-12}{E \cdot t_{\text {total }} \cdot w_{i}{ }^{3}}\left[\beta\left(g_{i}, w_{i}, w_{i+1}\right) \cdot \delta_{i+1}(x)\right. \\
& \quad-\left(\beta\left(g_{i}, w_{i}, w_{i+1}\right)+\beta\left(g_{i-1}, w_{i-1}, w_{i}\right)\right) \delta_{i}(x) \\
& \left.\quad+\beta\left(g_{i-1}, w_{i-1}, w_{i}\right) \cdot \delta_{i-1}(x)\right] \\
& \quad+\frac{12}{E \cdot t_{\text {total }} \cdot w_{i}{ }^{3}}\left[\alpha\left(g_{i}, w_{i}, w_{i+1}\right)-\alpha\left(g_{i-1}, w_{i-1}, w_{i}\right)\right]
\end{aligned}
$$

and

$$
\left\{\begin{aligned}
\frac{d^{4} \delta_{1}}{d x^{4}}(x)= & \frac{-12}{E \cdot t_{\text {total }} \cdot w_{1}{ }^{3}}\left[\beta\left(g_{1}, w_{1}, w_{2}\right) \cdot\left(\delta_{2}(x)-\delta_{1}(x)\right)\right. \\
\frac{d^{4} \delta_{N}}{d x^{4}}(x)= & \frac{12}{E \cdot t_{\text {total }} \cdot w_{N}{ }^{3}}\left[\beta\left(g_{1}, w_{2}\right)\right] \\
& \left.\cdot\left(\delta_{N}(x)-\delta_{N-1}(x)\right)-\alpha\left(g_{N-1}, w_{N-1}, w_{N}\right)\right] .
\end{aligned}\right.
$$

Therefore, we can express the bending equations as the following system:

$$
\frac{d^{4}}{d x^{4}} \underline{\delta}(x)=A \cdot \underline{\delta}(x)+\underline{b}
$$

where $\underline{\delta}(x)$ is the deflection vector, $A$ is a tridiagonal matrix and $\underline{b}$ is a column vector, both defined by the above equations. 
The boundary conditions for this differential system are fixedfixed

$$
\delta_{i}(0)=\delta_{i}(L)=0 ; \quad \frac{d \delta_{i}}{d x}(0)=\frac{d \delta_{i}}{d x}(L)=0 .
$$

To solve this system, the $N \times N$ matrix $A$ is diagonalized and the system is transformed to eigen-space. Because the coefficients of $A$ are dependent on the physical parameters of the different grating beams and are not generally correlated, this diagonalization is made numerically. $A$ is written as $P^{-1} D P$ where $D$ is the diagonal matrix of $A$ 's eigenvalues $\lambda_{i}$ and $P$ is the transformation matrix that transfers the "real" beams to the eigenvectors ("eigen-beams") space: $\underline{\delta}^{\prime}(x)=$ $P \cdot \underline{\delta}(x)$. After transforming $\underline{b}$ similarly $\left(\underline{b}^{\prime}=P \cdot \underline{b}\right)$, the system of equations is decoupled and each eigen-beam $i$ is governed by

$$
\frac{d^{4}}{d x^{4}} \delta_{i}{ }^{\prime}(x)=\lambda_{i} \cdot \delta_{i}{ }^{\prime}(x)+b_{i}^{\prime} .
$$

The solution of this equation has the following form:

$$
\begin{gathered}
\delta_{i}^{\prime}(x)=a_{1} \cosh \left(\lambda_{i}^{\frac{1}{4}} x\right)+a_{2} \sinh \left(\lambda_{i}^{\frac{1}{4}} x\right) \\
+a_{3} \cos \left(\lambda_{i}^{\frac{1}{4}} x\right)+a_{4} \sin \left(\lambda_{i}^{\frac{1}{4}} x\right)-\frac{b_{i}^{\prime}}{\lambda_{i}} .
\end{gathered}
$$

The coefficients $a_{i}$ are determined by the boundary conditions which have not been affected by the change of basis

$$
\delta_{i}{ }^{\prime}(0)=\delta_{i}{ }^{\prime}(L)=0 \quad \frac{d \delta_{i}{ }^{\prime}}{d x}(0)=\frac{d \delta_{i}{ }^{\prime}}{d x}(L)=0 .
$$

The coefficients $a_{i}$ must therefore verify the following system:

$$
\begin{aligned}
& \left(\begin{array}{lll}
\cosh \left(\lambda_{i}^{\frac{1}{4}} L\right)-\cos \left(\lambda_{i}^{\frac{1}{4}} L\right) \sinh \left(\lambda_{i}^{\frac{1}{4}} L\right)-\sin \left(\lambda_{i}^{\frac{1}{4}} L\right) & 0 & 0 \\
\sinh \left(\lambda_{i}^{\frac{1}{4}} L\right)+\sin \left(\lambda_{i}^{\frac{1}{4}} L\right) & \cosh \left(\lambda_{i}^{\frac{1}{4}} L\right)-\cos \left(\lambda_{i}^{\frac{1}{4}} L\right) & 0 \\
1 & 0 & 10 \\
0 & 1 & 0
\end{array}\right) \\
& \left(\begin{array}{l}
a_{1} \\
a_{2} \\
a_{3} \\
a_{4}
\end{array}\right) \\
& =\left(\begin{array}{r}
\frac{b_{i}^{\prime}}{\lambda_{i}}\left(1-\cos \left(\lambda_{i}^{\frac{1}{4}} L\right)\right) \\
\frac{b_{i}^{\prime}}{\lambda_{i}}\left(\sin \left(\lambda_{i}^{\frac{1}{4}} L\right)\right) \\
\frac{b_{i}^{\prime}}{\lambda_{i}} \\
0
\end{array}\right) .
\end{aligned}
$$

This system has a unique solution if its determinant is nonzero, which can be simplified to the following condition:

$$
\cosh \left(\lambda_{i}^{\frac{1}{4}} L\right) \cos \left(\lambda_{i}^{\frac{1}{4}} L\right) \neq 1 .
$$

If the product equals one, the magnetic force and the elasticity as they are modeled here cancel each other for any deflection. In reality, the inequality condition $\cosh \left(\lambda_{i}^{\frac{1}{4}} L\right) \cos \left(\lambda_{i}^{\frac{1}{4}} L\right)<1$ is used, as values of $\cosh \left(\lambda_{i}^{\frac{1}{4}} L\right) \cos \left(\lambda_{i}^{\frac{1}{4}} L\right)$ that are strictly greater than one lead to nonphysical solutions (negative deflections). Numerically, this condition is approximated by

$$
\lambda_{\max }{ }^{\frac{1}{4}} L<4.73 .
$$

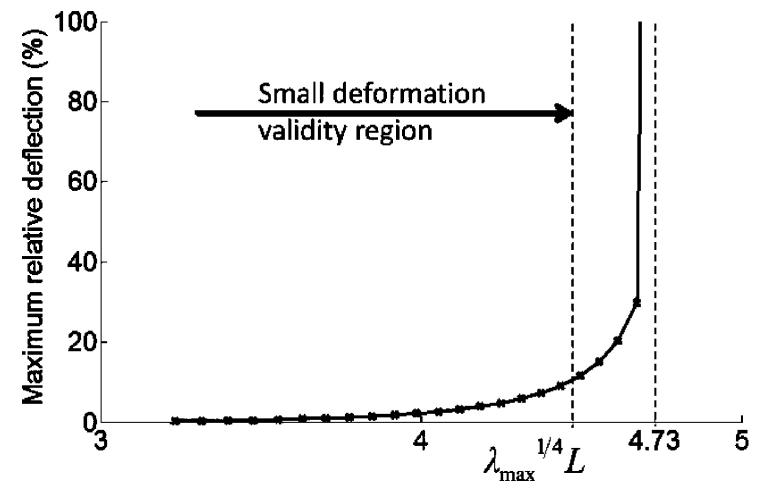

Fig. 12. Numerical simulation of the maximum relative deflection of the beams versus $\lambda_{\max }{ }^{1 / 4} L$ in a $1 \mu \mathrm{m}$ period, $50 \%$ duty-cycle, 100-beam grating. Nickel magnetic coating thicknesses range from $10 \mathrm{~nm}$ to $21.5 \mathrm{~nm}$ in $0.5 \mathrm{~nm}$ increments (cross markers).

However, one should avoid being too close to the equality condition, as the closer $\lambda_{i}^{\frac{1}{4}} L$ gets to 4.73 , the more significant is the deflection of beam $i$, invalidating the linearization of the magnetic force. Numerical simulations have shown that with $\lambda_{i}^{\frac{1}{4}} L$ up to 4.5 , the deflections still stay within a tolerable range, as shown In Fig. 12. Hence, we can replace (16) with

$$
\lambda_{\max }{ }^{\frac{1}{4}} L<4.5
$$

which is the nondimensional length that results in $10 \%$ relative deformation.

This restriction may be relaxed by shaping the load acting on the beams, e.g., by concentrating stronger magnets near the beam edges.

Since the eigenvalues are proportional to the magnetization $S^{2},(17)$ expresses the same limit as the critical magnetic layer strength condition that has been introduced in (10). In the special case of a uniform duty cycle grating $\left(w_{1}=w_{2}=w\right)$, the eigenvalues may be obtained analytically and the critical magnetization shown to be in approximate agreement with (10).

If (16) is satisfied, the $a_{i}$ s are

$$
\begin{aligned}
a_{1}= & \frac{1}{2\left(1-\cosh \left(\lambda_{i}^{\frac{1}{4}} L\right) \cos \left(\lambda_{i}^{\frac{1}{4}} L\right)\right)} \frac{b_{i}^{\prime}}{\lambda_{i}} \\
& \cdot\left(1+\cosh \left(\lambda_{i}^{\frac{1}{4}} L\right)\right)-\cos \left(\lambda_{i}^{\frac{1}{4}} L\right) \\
& -\cosh \left(\lambda_{i}^{\frac{1}{4}} L\right) \cos \left(\lambda_{i}^{\frac{1}{4}} L\right)-\sinh \left(\lambda_{i}^{\frac{1}{4}} L\right) \\
& \sin \left(\lambda_{i}^{\frac{1}{4}} L\right) \\
a_{2}= & \frac{1}{2\left(1-\cosh \left(\lambda_{i}^{\frac{1}{4}} L\right) \cos \left(\lambda_{i}^{\frac{1}{4}} L\right)\right)} \frac{b_{i}^{\prime}}{\lambda_{i}} \\
& \cdot\left(\cosh \left(\lambda_{i}^{\frac{1}{4}} L\right) \sin \left(\lambda_{i}^{\frac{1}{4}} L\right)+\cos \left(\lambda_{i}^{\frac{1}{4}} L\right) \sinh \left(\lambda_{i}^{\frac{1}{4}} L\right)\right. \\
& \left.\left.-\sinh \left(\lambda_{i}^{\frac{1}{4}} L\right)\right)-\sin \left(\lambda_{i}^{\frac{1}{4}} L\right)\right) \\
a_{3}= & \frac{b_{i}^{\prime}}{\lambda_{i}}-a_{1} \text { and } \\
a_{4}= & -a_{2} .
\end{aligned}
$$

The eigenbeam deflections are determined by substituting these coefficients in (15). By transferring back these solutions 


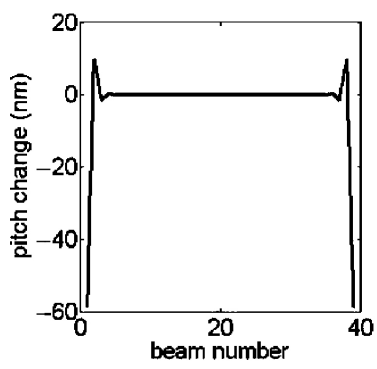

(a) (b)

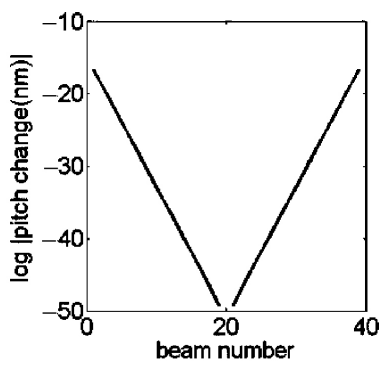

Fig. 13. (a) Simulation results of the change in grating pitch after actuation for a $50 \%$ duty cycle, constant period, 40-beam grating with $15 \mathrm{~nm}$ of nickel coating. (b) Logarithmic plot of the absolute value of pitch change showing the decay rate of the exponential envelope.

into the real beam space through $\underline{\delta}(x)=P^{-1} \cdot \underline{\delta}^{\prime}(x)$, the shape of every grating beam along the $x$ direction is obtained.

Simulation: The properties of the silicon nitride layer (Young's modulus of $300 \mathrm{GPa}$, thickness of $500 \mathrm{~nm}$ ) and the length of the grating beams $(250 \mu \mathrm{m})$ are parameters that are maintained for all the simulations. The magnetic material used is nickel, assumed to be magnetically saturated $\left(B_{s}=0.6 \mathrm{~T}\right)$ in the perpendicular direction. In practice, such a magnetization of the nickel is not easily achieved and we might conservatively consider the force obtained by simulation to be higher than the actual.

A grating with constant pitch and duty cycle, such as one easily fabricated through interference lithography can theoretically not be actuated. The total magnetic force on one grating beam under actuation will be zero since the interactions with the two neighboring beams cancel each other. This uninteresting stable position can be broken with boundary conditions. If we remove a few beams at the edges of the grating, the side beams do not have this equilibrium at no deflection and will bend toward the middle of the grating, generating a cascading actuation throughout the other grating beams.

Computing the shape of the grating for this setup, we observe that only the side beams bend notably. As described in the simulation results (Fig. 13), the deflections alternatively change sign and their magnitudes decrease within an exponential envelope, which could be used to create exponentially chirped periodic structures.

The exponential decay rate is dependent only on the parameters of the magnetic coating and not on the number of beams as showed in Fig. 14. Additionally, one might note that the sign alternation of the deflections predicts a pairing of the beams by two-by-two clumping should the magnetic coating strengthen.

For this particular grating, (10) yields a first approximation of critical nickel coating thickness of $12 \mathrm{~nm}$. Also, as this is a particular case where the eigenvalues of the $A$ matrix are known, the more rigorous (17) can be applied and gives a critical thickness of $21 \mathrm{~nm}$.

Other distributions of width and interbeam gaps are investigated to get other interesting actuation profiles. The grating period is kept constant and only the duty cycle (ratio of beam

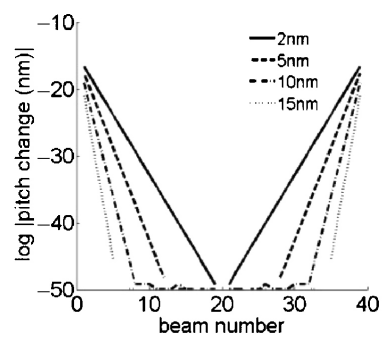

(a)

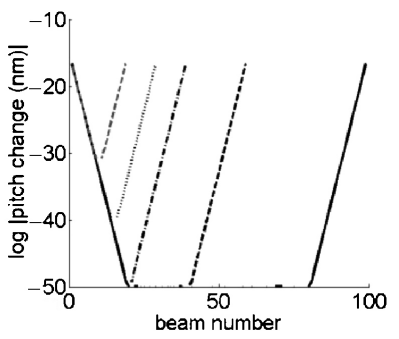

(b)
Fig. 14. Exponential decay rate of the pitch change envelope as a function (a) of nickel coating thickness and (b) of the number of beams for a $15 \mathrm{~nm}$ nickel coating.

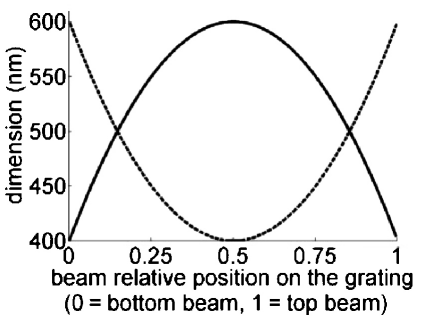

Fig. 15. Grating duty cycle parabolic distribution with a peak-to-peak amplitude of $40 \%$. Both cases were the plain curve and the dotted curve alternatively represent the width distribution and the gap distribution are studied.

width over period) is tuned along the different beams to maintain a similar diffraction pattern. Initially, a simple symmetric linear profile of duty cycle distribution is implemented (Vshape). However, the actuation profile does not produce any noteworthy pattern and the discontinuity of the distribution in the middle of the grating (bottom of the V-groove) creates a disturbance.

Next a parabolic distribution of duty cycle is tested. Both cases, where the beams become narrower or wider at the center, are investigated (Fig. 15). They turn out to produce similar actuated pattern. In each case, side effects as described for the constant duty cycle grating occur and at the edges we observe the same alternating pitch change with exponential decay. However, an interesting pattern is produced in the middle region where side effects have faded out and become negligible.

The bulk of the grating (beams subjected to side effects are removed) has a pitch variation shaped similarly to a cowboy hat. Its central part (about $50 \%$ of the grating) fits a parabola, as shown Fig. 16. This central portion is dominant if the grating has a small number of beams or if the amplitude of the duty cycle distribution is small. Further analysis of the plot has determined that the profiles actually fit hyperbolic cosine curves even better over a wider range. However, the parabola shape draws more interest for certain applications such as aperiodic photonic structures like the tunable GRIN lens presented in Fig. 3.

It should be mentioned that the same effect could be produced by changing the lengths of the grating beams instead of the width and the gap in order to keep the duty cycle constant. Indeed, (6) indicates that the rigidity of the beam approximately goes as $w^{3} / L^{4}$. So changing $L$ accordingly, the same effect as changing $w$ should be produced, even though 


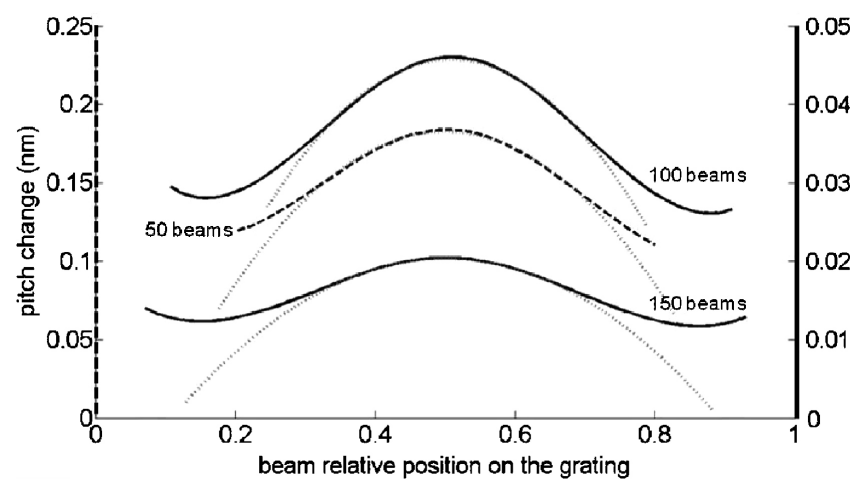

Fig. 16. Simulation of the change in pitch after actuation for gratings with 50,100 , and 150 beams. The central parts of the curves are fitted to parabolas drawn in dotted grey. The amplitude of the parabolic distribution of width and gaps is $40 \%$ with the thicker beams at the edges. The magnetic coating is made of $15 \mathrm{~nm}$ nickel.

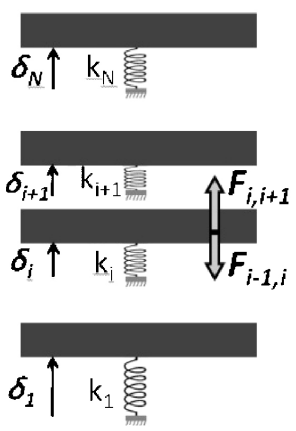

Fig. 17. 1-D model of the grating.

changes in the magnetic force would also need to be taken into account.

Additionally, shaping the magnetic coating into nanomagnets (as in Fig. 8) and varying their concentration along the beams could give another degree of freedom over the magnetic force. This would allow other shapes of distorted grating that can be useful for other applications and also add precision, flexibility, range and controllability over the actuation. For example, the parabolic trend seen in Fig. 16 may be useful for the lens-like structure of [25].

\section{F. Clumping Regime}

If the magnetic layer strength is higher than the thresholds defined in (17) and the simpler but more approximate (10), then any beam rigidity is overcome by the magnetic attraction provoking multiple beams to clump together. The magnetic force cannot be linearized anymore to model this phenomenon as the term $1 / \mathrm{g}$ is exploding when beams are in contact. Since the system to be solved has now $N$ nonlinear coupled equations, an analytical solution is discarded and a numerical energy minimization approach is taken. Firstly, a simplifying assumption is made where the grating is assumed to be a 1-D lumped model with rigid beams, as schematically represented in Fig. 17. The state of the grating remains represented by the vector $\underline{\delta}$, now independent of $x$, where each component $\underline{\delta_{i}}$ corresponds to the deflection of the $i$ th beam of the grating.
The total energy of a configuration consists of an elasticity term and a magnetic term. By using the beam stiffness defined in (6) by

$$
k_{i} \equiv \frac{32 \cdot E \cdot t_{\text {total }} \cdot w_{i}^{3}}{L^{4}}
$$

the elastic energy is written as

$$
\frac{1}{2} \sum_{i} k_{i} \cdot \delta_{i}=\frac{1}{2} \underline{k} \cdot \underline{\delta} .
$$

The magnetic interaction energy between two beams $i$ and $i+1$ is derived by taking the integral of the force expression in (2)

$$
\begin{array}{r}
E_{\mathrm{mag}_{-} i, i+1}=\frac{M^{2} \cdot t_{m}^{2}}{2 \pi \mu_{0}}\left(\ln \left(\left|g_{i}\right|\right)-\ln \left(\left|g_{i}+w_{i}\right|\right)\right. \\
\left.-\ln \left(\left|g_{i}+w_{i+1}\right|\right)+\ln \left(\left|g_{i}+w_{i}+w_{i+1}\right|\right)\right) .
\end{array}
$$

Additionally, our system is constrained by the fact that the beams cannot cross each other. When in contact, the beams are subject to a contact force that is not taken into account in (18). To correct for this and prevent the interbeam gap from becoming negative, we introduce an artificial energy barrier representing the contact force. The specific form of barrier used in our simulations was symmetric with respect to the energy profile in the physically meaningful regime and it was also smoothened to eliminate the singularity of the magnetic energy at zero gap. The symmetric magnetic energy is

$$
\begin{gathered}
E_{m a g_{-} i, i+1}=\frac{M^{2} \cdot t_{m}^{2}}{2 \pi \mu_{0}}\left(\ln \left(\left|g_{i}\right|\right)-\ln \left(\left|g_{i}\right|+w_{i}\right)\right. \\
\left.-\ln \left(\left|g_{i}\right|+w_{i+1}\right)+-\ln \left(\left|g_{i}\right|+w_{i}+w_{i+1}\right)\right) .
\end{gathered}
$$

This way having a negative gap will only increase the energy and the algorithm will avoid the negative gap while keeping a valid expression for positive gaps.

Furthermore, the magnetic interaction energy expression relies on a magnetic dipoles model [see (1)]. This assumption does not hold when the dipoles are close together, which happens when an interbeam gap tends to zero. The correct interaction force for two nearby magnetic surfaces is $F=$ $A \cdot B^{2} /\left(2 \mu_{0}\right)$, where $A$ is the area and $B$ the magnetic field at the surface. Assuming that the beam is infinitely long $\left(L>>t_{m}, w\right)$, which holds in our case where the aspect ratio is typically around 1:500, an analytical expression for the field $B$ can be derived from basic magnetostatics and an equivalent coil

$$
B=\frac{M}{\pi} \arctan \left(\frac{2 w}{t_{m}}\right) .
$$

The per unit length magnetic attraction when the grating beams are in proximity therefore equals

$$
F_{\text {lin_prox }}=\frac{t_{m} M^{2}}{2 \mu_{0} \pi^{2}} \arctan ^{2}\left(\frac{2 w}{t_{m}}\right) .
$$

A threshold gap $g_{t}$ can be determined for which $F_{\text {lin }}\left(g_{t}\right)$ as defined in (2) is equal to $F_{\text {lin_prox }}$. Hence, the actual magnetic 
force is piecewise defined as

$$
F_{\max }(g)=\left\{\begin{array}{l}
g \geq g_{t}: \\
\frac{M^{2} \cdot t_{m}^{2}}{2 \pi \mu_{0}}\left(\frac{1}{g}-\frac{1}{g+w_{1}}-\frac{1}{g+w_{2}}+\frac{1}{g+w_{1}+w_{2}}\right) \\
g \leq g_{t}: \\
\frac{t_{m} M^{2}}{2 \mu_{0} \pi^{2}} \arctan ^{2}\left(\frac{2 w}{t_{m}}\right) .
\end{array}\right.
$$

Note that the function $g \rightarrow F_{\text {mag }}(g)$ is continuous by definition of $g_{t}$.

The magnetic energy term then becomes

$$
E_{\text {mag } \_ \text {i }, i+1}=\left\{\begin{array}{l}
g_{i} \geq g_{t}: \\
\frac{M^{2} \cdot t_{m}^{2}}{2 \pi \mu_{0}}\left(\ln \left(\left|g_{i}\right|\right)-\ln \left(\left|g_{i}\right|+w_{i}\right)\right. \\
\left.\quad-\ln \left(\left|g_{i}\right|+w_{i+1}\right)+-\ln \left(\left|g_{i}\right|+w_{i}+w_{i+1}\right)\right) \\
g_{i} \leq g_{t}: \\
\frac{t_{m} M^{2}}{2 \mu_{0} \pi^{2}} \arctan ^{2}\left(\frac{2 w}{t_{m}}\right) \cdot\left(\left|g_{i}\right|-g_{t}\right) \\
+\frac{M^{2} \cdot t_{m}^{2}}{2 \pi \mu_{0}}\left(\ln \left(\left|g_{t}\right|\right)-\ln \left(\left|g_{t}\right|+w_{i}\right)-\ln \left(\left|g_{t}\right|+w_{i+1}\right)\right. \\
\left.+-\ln \left(\left|g_{t}\right|+w_{i}+w_{i+1}\right)\right) .
\end{array}\right.
$$

In that form, the energy term stays continuous at the threshold gap and keeps its symmetry. However, the energy well has a V-groove shape in the zero gap proximity. In order to enjoy nice continuity properties at the well bottom (typically $C^{1}$ ) for the optimization algorithm, this sharp tip is rounded parabolically under a critical gap $g_{c}$. To avoid any disturbance of this artifact over the validity range of the magnetic force, this critical gap is chosen to be negligibly small compared to $g_{t}$. Since $g_{t}$ is in the order of tens or hundreds of nanometers, $g_{c} \sim 1 \mathrm{~nm}$ is a safe choice.

By adding the elastic energy and each of the magnetic interaction energies, we obtain the total energy of the grating

$$
E_{\text {total }}=\frac{1}{2} \underline{k} \cdot \underline{\delta}+\sum_{i=1}^{N-1} E_{\text {mag } \_i, i+1} .
$$

The equilibrium of the grating beams is determined by finding the vector $\underline{\delta}$ that minimizes this total energy. The optimization algorithm presented here employs a fixed step gradient descent method, where the minimizing $\underline{\delta}$ is the converging value of the following sequence:

$$
\underline{\delta}_{n+1}=\underline{\delta}_{n}-\mu \cdot \underline{E}^{\prime}\left(\underline{\delta}_{n}\right) .
$$

As the algorithm starts from the configuration at rest, it reproduces exactly the trajectories of the grating beams. Vibrations or thermal agitation or other means for crossing energy barriers can lead to lower energy equilibria, but we did not consider such effects in this paper.

After this 1-D algorithm has determined the beams that bundle together, the shape of the beams is determined. Assuming that one beam is predicted to be touching a neighbor at a deflection $\delta_{m}$ as depicted in the schematics in Fig. 18,

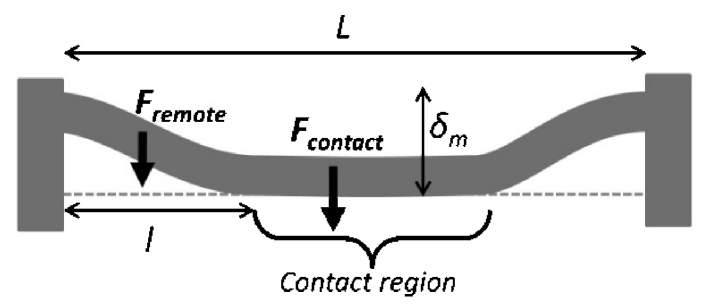

Fig. 18. Shape of a beam in contact with its neighbor.

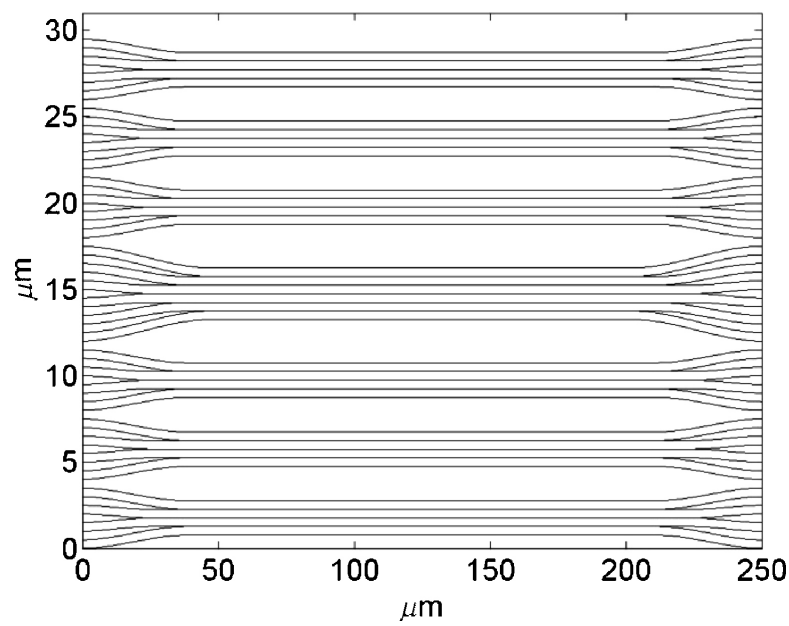

Fig. 19. Simulation result of the deformed shape of a 30-beam grating with $100 \mathrm{~nm}$ of nickel coating under actuation.

the deflection along the length $l$ is calculated using (11), the magnetic force at contact and the magnetic force at positive gap. For this calculation, the dependence of the latter to the gap is neglected. This assumption is based on the fact that the variations of this force with the gap are small compared to the contact force. The constant value chosen for this remote magnetic force is the average force over the distance $l$.

The value of $l$ is determined by first calculating the shape of a beam with length $l$, fixed-fixed boundary conditions $\left[\delta(0)=0, \delta(l)=-\delta_{m}, \delta^{\prime}(0)=0, \delta^{\prime}(l)=0\right]$ and subjected to a uniform load $F_{\text {remote }}$. The unknown $l$ is then determined by matching the contact force to the reaction force required to bend the beam at $l$. This actually involves solving a fourth order polynomial equation that can have up multiple physically acceptable solutions. Fortunately in that case, common sense can retain only one of them after displaying the grating shape for the different solutions.

Simulation: The simulation in this case uses the same physical parameters as for the small deformation case. However, the grating magnetic thickness is not restricted. The actuation of conventional gratings ( $50 \%$ duty cycle) with different numbers of beams and different coating thicknesses are simulated (Fig. 20). The thin coating cases $(10-20 \mathrm{~nm})$ confirm the critical thickness that has been calculated and simulated earlier to about $21 \mathrm{~nm}$, as the two-by-two clumping starts at about $20 \mathrm{~nm}$. 


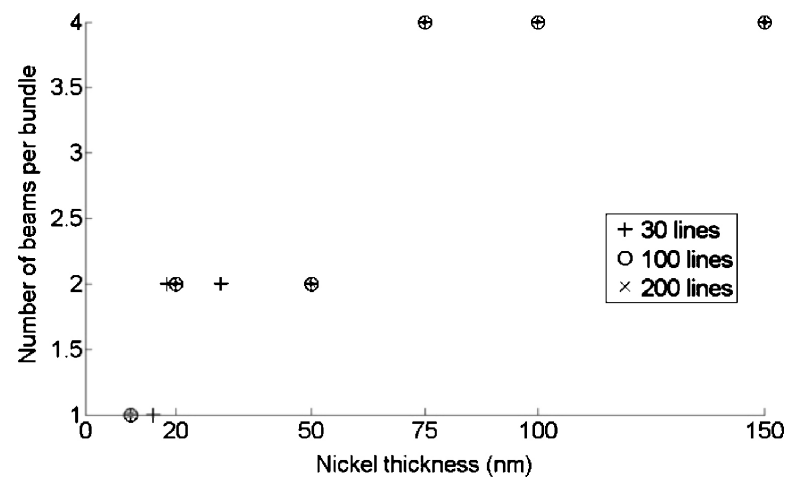

Fig. 20. Number of beams clumping together versus thickness of the nickel coating for 30-, 100-, and 200-beam gratings.

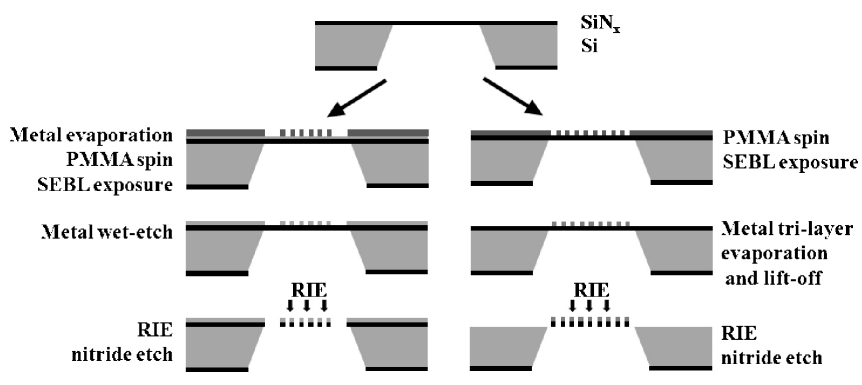

Fig. 21. Microfabrication process presenting both wet-etch and lift-off metal patterning methods.

\section{FABRICATION PROCESS}

Before the actual patterning of the grating, the microfabrication process (Fig. 21) starts with the formation of a silicon nitride membrane. First, $500 \mathrm{~nm}$ of low stress silicon nitride layer is deposited on both side of a silicon wafer by low pressure chemical vapor deposition. The backside layer is patterned using standard photolithography and dry etching processes. The nitride layer acts then as a hard mask for the anisotropic etching of the bulk silicon in a Potassium Hydroxide $(\mathrm{KOH})$ bath, releasing a silicon nitride membrane on the top side. Next, two different ways of patterning the magnetic coating into a grating have been carried out. For the first one, a nickel layer is uniformly evaporated on top of the membrane and the grating pattern is written by scanning electron beam lithography into a spin-coated electron sensitive resist film of poly(methyl methacrylate) (PMMA). After development in a bath of methyl isobutyl ketone diluted at $33 \%$ in isopropanol for $1 \mathrm{~min}$, the nickel is wet etched using transene thin-film-B type etchant. The resist is then stripped in an acetone bath. For the second method of metal patterning, the PMMA is spincoated directly onto the silicon nitride. Once patterned and developed, a tri-layer of metal $(\mathrm{Cr}, \mathrm{Co} / \mathrm{Ni}$, and $\mathrm{Cr})$ is evaporated in an electron beam evaporator. The resist is then lifted off in a hot bath of n-methylpyrrolidone revealing the metal grating pattern. This lift-off method has the advantage that it allows the formation of a multilayer metal coatings without the undercut produced by anisotropic wet etching techniques. The chromium bottom layer $(8 \mathrm{~nm})$ role is to promote adhesion to the nitride whereas the top layer $(15 \mathrm{~nm})$ provides oxidization protection and acts as a hard mask for the subsequent etch step.

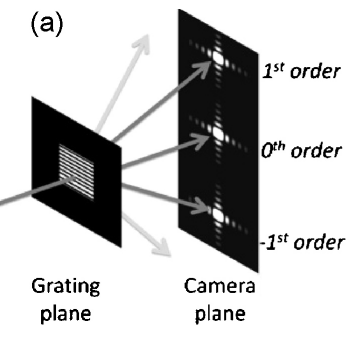

(b)

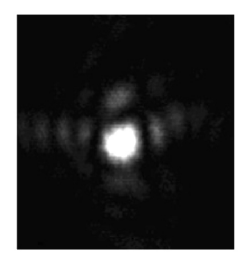

(c)

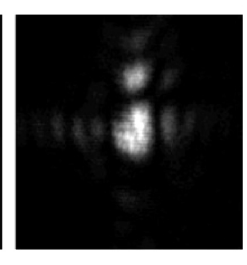

Fig. 22. (a) Schematic of the diffraction pattern from a grating in a square window used in transmission. (b) Experimental image of the first order without external magnetic field. (c) Under external magnetic field.

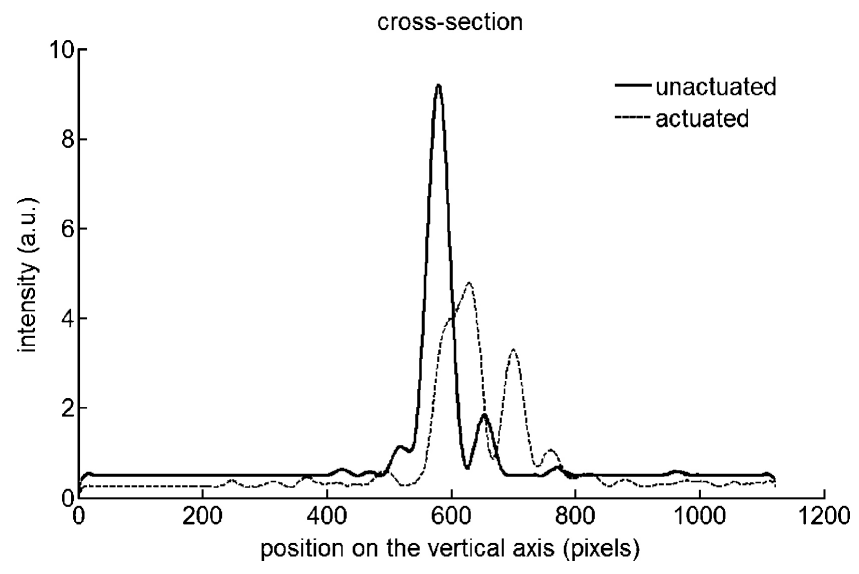

Fig. 23. Intensity cross-section of the first diffraction order for the actuated and unactuated grating. The pixel size is $5 \mu \mathrm{m}$.

The nature and amount of magnetic material between the two chromium layers is chosen to have the desired magnetization and strength properties. For both methods, the silicon nitride that is left uncovered is subsequently etched away by a reactive ion etch process, releasing freestanding grating beams. To be self-supported, these beams are patterned longer than the width of the membrane.

\section{EXPERIMENTAL RESULTS}

\section{A. Small Deformations}

In an initial experiment, the grating was $200 \mu \mathrm{m}$ long, had a pitch of $800 \mathrm{~nm}$ and $15 \mathrm{~nm}$ of nickel coating patterned by the wet-etch method. It was then illuminated at normal incidence by an expanded doubled Nd:YAG laser beam $(\lambda=532 \mathrm{~nm})$. The transmitted diffraction pattern was then observed at the Fourier plane of a collector lens. Next to the grating, a neodymium permanent magnet with $0.5 \mathrm{~T}$ of field strength at its surface was mounted on a translation stage. The magnetic field was oriented perpendicular to the grating beams.

Without the external magnetic field, we observed the typical diffraction pattern resulting from a square grating as depicted schematically in Fig. 22(a). The different orders of diffraction are due to the grating and the 2-D squared sine cardinal patterns are due to the aperture window. The square pattern observed at the first order of diffraction is shown in Fig. 22(b). When the permanent magnet was brought to the proximity of the grating, the diffraction pattern changed, indicating a 


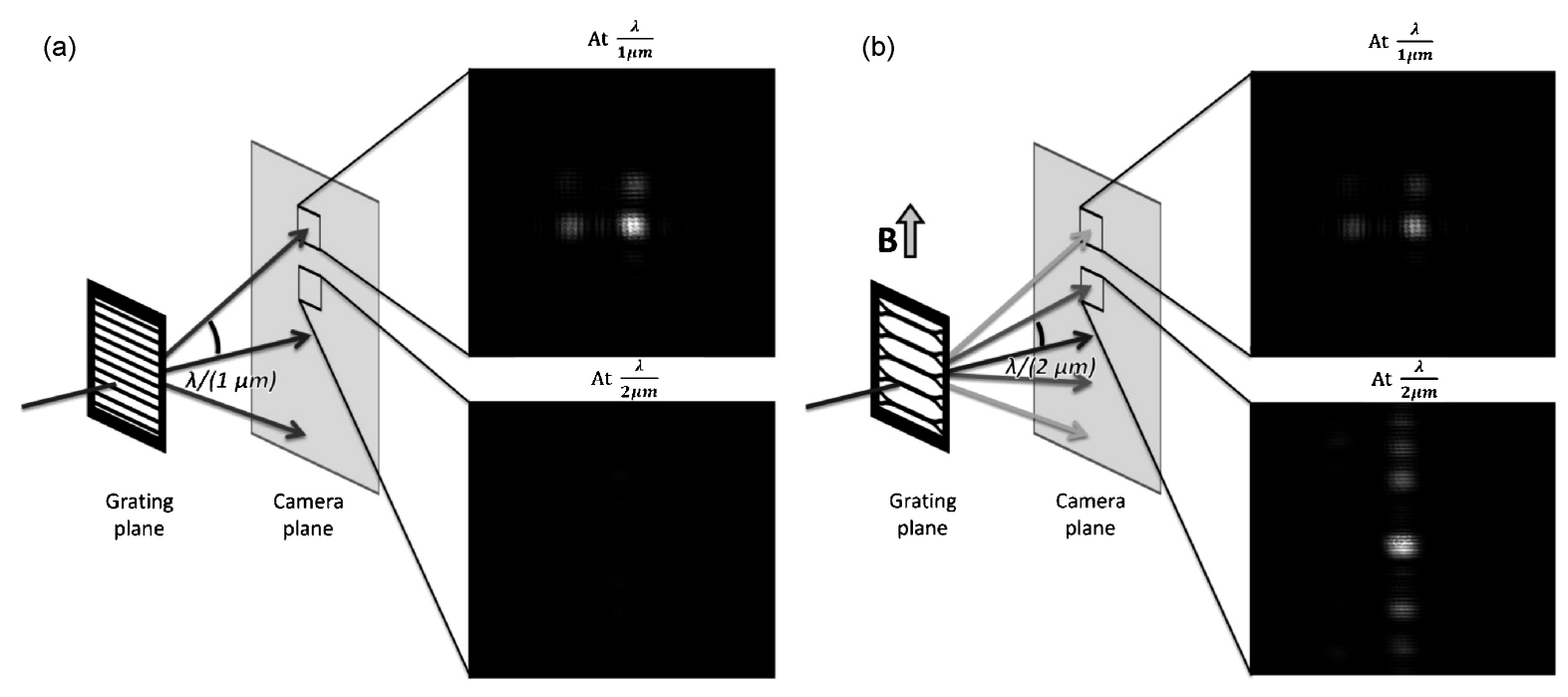

Fig. 24. Schematic setup of the clumping experiment and pictures of the same sample taken under identical experimental conditions at angles of $\lambda / 1 \mu$ m and $\lambda / 2 \mu \mathrm{m}$ (a) without external magnetic field and (b) under external magnetic field.
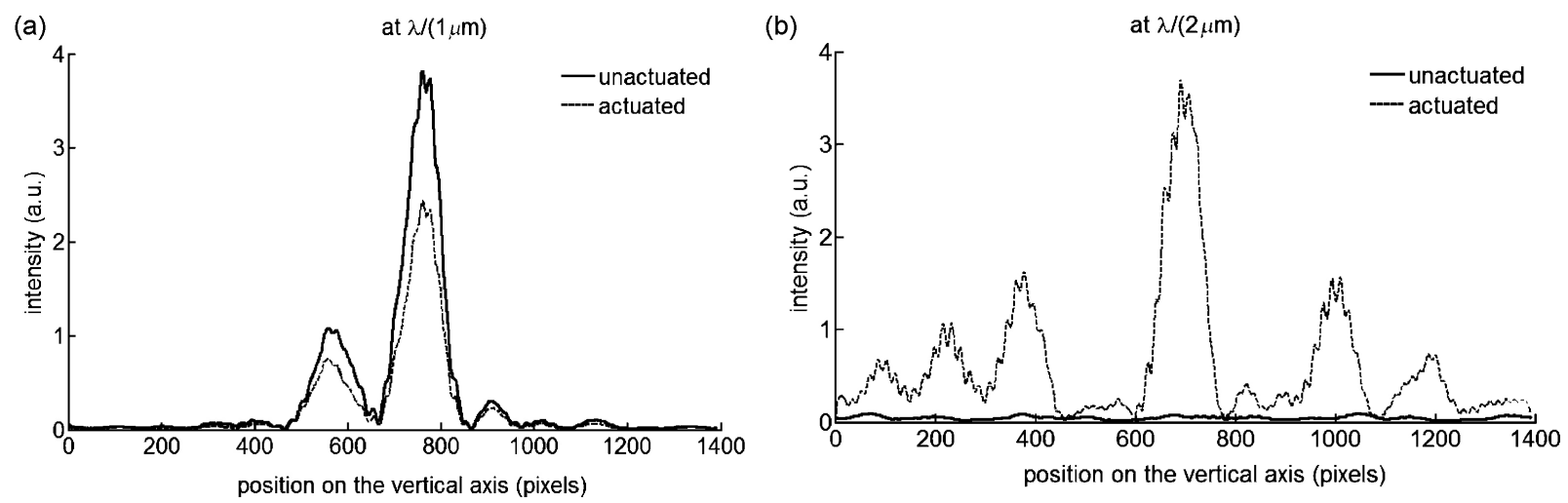

Fig. 25. Intensity cross-sections of the diffraction orders at angles of (a) $\lambda / 1 \mu \mathrm{m}$ and (b) $\lambda / 2 \mu \mathrm{m}$ for the actuated and unactuated cases. The pixel size is 5 $\mu \mathrm{m}$.

modification of the grating pitch. As shown in Fig. 22(c), the first order of diffraction shifted and smeared. The shifting was due to the over-all compression of the grating and the smearing to the nonuniformity of the pitch variation after actuation. Quantitatively, the displacement of the first diffraction order was about $200 \mu \mathrm{m}$ (Fig. 23), which corresponded to a $0.7 \mathrm{mrad}$ angle displacement, as the distance to the camera plane was $430 \mathrm{~mm}$. This was consistent with the simulation that expected a mean pitch variation of $0.72 \mathrm{~nm}$ (angular shift of $0.6 \mathrm{mrad})$.

\section{B. Clumping Regime}

The grating tested in this experiment was $250 \mu \mathrm{m}$ long and had a $1 \mu \mathrm{m}$ period. To test the clumping regime of the magnetic actuation, a stronger magnetic coating of $120 \mathrm{~nm}$ cobalt was deposited. A sample containing four gratings fabricated simultaneously using the lift-off method of Section IV was placed in the same optical setup as in the initial experiment. The illumination beam was wide enough to encompass all four gratings, creating high frequency modulations in the diffraction pattern. As in the previous experiment, the gratings operate in transmission. Without external magnetic field, we observed a classic diffraction pattern from a regular grating showing first orders of diffraction at an angle of $\lambda /(1 \mu \mathrm{m})$ as displayed in Fig. 24(a). However, some imperfect experimental conditions make the bottom and right-hand side lobes seam dimmer. When the external magnetic field was applied, the former first orders became dimmer and new orders of diffraction at half the angle $\lambda /(2 \mu \mathrm{m})$ appeared [Fig. 24(b)]. This pattern corresponds to the diffraction of a $2 \mu \mathrm{m}$ period grating, signifying that the grating beams clumped two by two.

As the grating had a duty cycle of $50 \%$, the second order of diffraction should have theoretically been missing, which predicted an extinction of the $\lambda /(1 \mu \mathrm{m})$ order under full actuation. However, the drop of intensity measured from the cross-sections in Fig. 25 was not total. This is because of residual diffraction from unclumped beams in the neighboring gratings.

\section{CONCLUSION}

We presented a novel method to actuate diffractive optical elements using inter-nanomagnet forces. This actuation combines the advantages of both analog and digital tuning techniques. A magnetically coated grating under small magnetic field or having weak magnetic properties deforms slightly and 
enables fine analog tuning of the pitch. Gratings with stronger coating or under more intense field undergo a clumping of their beams effectively permitting a discrete steps "digital" tuning of the grating pitch. Theoretical analysis and linear and nonlinear numerical simulations have been carried out establishing with good agreement of the critical parameters characterizing the transition between the two regimes. Furthermore, the simulations showed interesting grating pitch distribution profiles under actuation such as a decaying exponential for a regular grating or a parabola for a grating with a parabolic dutycycle distribution. Also, these simulations can be used to determine the 2-D shape under magnetic actuation of virtually any periodic structures with variable parameters (period, thickness, duty-cycle, coating). Experimentally, both the analog and digital regimes have been observed through examination of the diffraction pattern of different fabricated gratings. A grating coated with a $20 \mathrm{~nm}$ nickel layer has shown a continuous average pitch reduction of up to $0.1 \%$ under a magnetic field increasing up to $0.1 \mathrm{~T}$. Another grating coated with a stronger magnetic layer of $120 \mathrm{~nm}$ cobalt has demonstrated an abrupt period doubling signifying a two-by-two clumping of the grating beams. These results have proven the concept of diffraction grating actuation through inter-nanomagnet forces. This technique provides both analog and digital tuning by simply adding a magnetic coating to existing gratings without the need for other actuation features on the sample such as space consuming electrostatic comb-drives, piezo or thermal actuators. However, it still lacks the precision, range, and particularly the tuning control offered by existing methods. Additionally, even though the magnetic actuation allows interesting shapes, they require complex parameters distributions and other profiles of interest such as a simple linear variation of the grating pitch are not achievable yet with this technique. In a future work, the magnetic coating will be shaped into patterned vertical nanomagnets that should minimize these drawbacks. Nevertheless, this is a promising step toward the goal of an integrated tunable optical microsystem actuated by nanomagnets.

\section{ACKNOWLEDGMENT}

The authors would like to thank M. Mondol and J. Daley for experimental assistance and the anonymous reviewers for their valuable suggestions. The structures were fabricated at the Massachusetts Institute of Technology (MIT) Scanning Electron Beam Lithography Facility and the MIT Nanostructures Laboratory.

\section{REFERENCES}

[1] K. Saruta, M. Nishida, M. Yamaguchi, Y. Ito, K. Yamashita, A. Taguchi, K. Oniki, and H. Tamada, "Ultrahigh contrast $\mathrm{GxL}^{\mathrm{TM}}$ device for laser projector," in Proc. Int. Conf. Solid-State Sens. Actuators Microsyst. (Transducers), 2007, pp. 2135-2138.

[2] Y. Ito, K. Sarutaa, H. Kasaia, M. Nishidaa, M. Yamaguchia, K. Yamashitaa, A. Taguchib, K. Onikib, and H. Tamadab, "Highperformance blazed $\mathrm{GxL}^{\mathrm{TM}}$ device for large-area laser projector," in Proc. 4th Int. Soc. Opt. Eng., Micro-Opto-Electro-Mech Syst. Display Imag. Miniaturized Microsyst., vol. 6114. 2006, pp. $1-12$.
[3] M. B. Sinclair, M. A. Butler, A. J. Ricco, and S. D. Senturia, "Synthetic spectra: A tool for correlation spectroscopy," Appl. Opt., vol. 36, no. 15, pp. 3342-3348, 1997.

[4] M. Tormen, Y.-A. Peter, P. Niedermann, A. Hoogerwerf, H. Shea, and R. Stanley, "Deformable MEMS grating for wide tunability and high operating speed," in Proc. 4th Int. Soc. Opt. Eng., Micro-Opto-ElectroMech. Syst. Display, Imag. Miniaturized Microsyst., vol. 6114. Jan. 2006, pp. 61140C-1-61140C-11.

[5] A. Pothisorn and A. J. Hariz, "Deformable grating light modulator array for use as wavelength-selective switch," in Proc. Int. Soc. Opt. Eng. 3rd Micro-Nanotechnol.: Mater. Process. Packag. Syst., vol. 6415. Dec. 2006, p. $64151 \mathrm{~N}$

[6] O. Solgaard, F. S. A. Sandejas, and D. M. Bloom, "Deformable grating optical modulator," Opt. Lett., vol. 17, no. 9, pp. 688-690, 1992.

[7] C. W. Wong, Y. Jeon, G. Barbastathis, and S. G. Kim, "Analog piezoelectric-driven tunable gratings with nanometer resolution," $J$. Microelectromech. Syst., vol. 13, no. 6, pp. 998-1005, Dec. 2004.

[8] W.-C. Shih, C. W. Wong, Y. B. Jeon, S.-G. Kim, and G. Barbastathis, "MEMS tunable gratings with analog actuation," Inf. Sci., vol. 149, no. $1-3$, pp. $31-40$, Jan. 2003

[9] W.-C. Shih, S. G. Kim, and G. Barbastathis, "High-resolution electrostatic analog tunable grating with a single-mask fabrication process," $J$. Microelectromech. Syst., vol. 15, no. 4, pp. 763-769, Aug. 2006.

[10] M. Tormen, Y.-A. Peter, P. Niedermann, A. Hoogerwerf, and R. Stanley, "Deformable MEMS grating for wide tunability and high operating speed," J. Opt. A Pure Appl. Opt., vol. 8, no. 7, pp. S337-S340, 2006.

[11] W.-C. Shih, C. W. Wong, Y. B. Jeon, S.-G. Kim, and G. Barbastathis, "Electrostatic and piezoelectric analog tunable diffractive gratings," in Proc. Conf. Lasers Electro-Opt., May 2002, pp. 21-26.

[12] S. C. Truxal, Y.-C. Tung, and K. Kurabayashi, "A flexible nanograting integrated onto silicon micromachines by soft lithographic replica molding and assembly," J. Microelectromech. Syst., vol. 17, no. 2, pp. 393-401, Apr. 2008.

[13] X. M. Zhang and A. Q. Liu, "A MEMS pitch-tunable grating add/drop multiplexers," in Proc. IEEE/Lasers Electro-Opt. Soc. Int. Conf. Opt. Micro-Electromech. Syst., Aug. 2000, pp. 21-24.

[14] Y.-S. Yang, Y.-H. Lin, Y.-C. Hu, and C.-H. Liu, "A large-displacement thermal actuator designed for MEMS pitch-tunable grating," $J$. Micromech. Microeng., vol. 19, no. 1, pp. 15001-15012, 2009.

[15] X. M. Zhang, Q. W. Zhao, T. Zhong, A. B. Yu, E. H. Khoo, C. Lu, and A. Q. Liu, "Variable nano-grating for tunable filters," in Proc. Int. Conf. Solid-State Sens. Actuators Microsyst. (Transducer), Jun. 2007, pp. 2417-2420.

[16] D. M. Burns and V. M. Bright, "Development of microelectromechanical variable blaze gratings," Sens. Actuators A Phys., vol. 64, no. 1, pp. 715, Jan. 1998.

[17] Y.-T. Yu, W.-Z. Yuan, and D.-Y. Qiao, "Electromechanical characterization of a new micro programmable blazed grating by laser Doppler vibrometry," Microsyst. Technol., vol. 15, no. 5, pp. 853-858, 2009.

[18] H. Mavoori, S. Jin, R. P. Espindola, and T. A. Strasser, "Enhanced thermal and magnetic actuations for broad-range tuning of fiber Bragg grating-based reconfigurable add-drop devices," Opt. Lett., vol. 24, no. 11 , pp. 714-716, 1999.

[19] A. Nichol, W. Arora, and G. Barbastathis, "Thin membrane selfalignment using nanomagnets for 3-D nanomanufacturing," J. Vacuum Sci. Technol. B, vol. 24, no. 6, p. 3128, Nov.-Dec. 2006.

[20] A. Nichol, M. Deterre, and G. Barbastathis, "Stretching and alignment of compliant nanomembranes by embedded nanomagnets," in Proc. 53rd Int. Conf. Electron Ion Photon Beam Technol. Nanofabrication, May 2009, Paper 7A.3.

[21] J. B. Pendry, D. Schurig, and D. R. Smith, "Controlling electromagnetic fields," Science, vol. 312, no. 5781, pp. 1780-1782, Jun. 2006.

[22] J. Valentine, S. Zhang, T. Zentgraf, E. Ulin-Avila, D. A. Genov, G. Bartal, and X. Zhang, "3-D optical metamaterial with a negative refractive index," Nature, vol. 455, no. 7211, pp. 376-379, Sep. 2008.

[23] J. Yao, Z. Liu, Y. Liu, Y. Wang, C. Sun, G. Bartal, A. M. Stacy, and X. Zhang, "Optical negative refraction in bulk metamaterials of nanowires," Science, vol. 321, no. 5891, p. 930, Aug. 2008.

[24] N. Kundtzl and D. R. Smith, "Extreme-angle broadband metamaterial lens," Nature Mater., vol. 9, pp. 129-132, Jan. 2010.

[25] S. Takahashi, P. Stellman, W. Arora, and G. Barbastathis, "Adiabatic focusing of light in subwavelength high-index contrast dielectrics," in Proc. Int. Symp. Nanomanuf., Nov. 2008, Paper 1B.3.

[26] F.-J. Ulm, A New Introduction to Engineering Mechanics. Cambridge, MA: MIT Press, 2009, ch. 5, pp. 175-223. 
[27] J. R. Barber, Intermediate Mechanics of Materials. New York: McGrawHill, 2001, ch. 1, pp. 17-19.

[28] S. D. Senturia, Microsystem Design. Norwell, MA: Kluwer Academic, 2001 , ch. 6 , pp. $125-145$

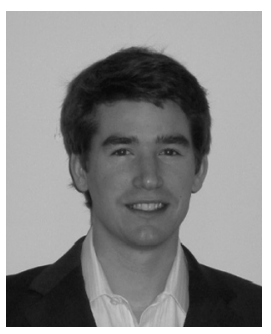

Martin Deterre received the Engineer degree from the Ecole Polytechnique, Palaiseau, France, in 2008, and is currently working toward the M.S. degree in mechanical engineering from the Department of Mechanical Engineering, Massachusetts Institute of Technology, Cambridge.

His current research interests include microelectromechanical systems, tunable optical devices, nanomembranes technology, and 3-D assembly of nanostructures.

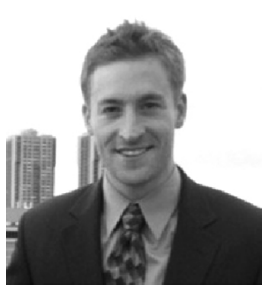

Anthony J. Nichol received the B.S. degree in mechanical engineering from the University of Wisconsin, MD, in 2004. He is currently working toward the $\mathrm{Ph} . \mathrm{D}$. degree in mechanical engineering from the Massachusetts Institute of Technology, Cambridge, with a focus on the use of nanomagnets for alignment and actuation of nanostructures.

He co-founded and is currently the Chief Technical Officer with FLEx Lighting, LLC, a start-up company out of Chicago, IL, that has developed a proprietary film light emitting diode lighting system. As a National Science Foundation Graduate Fellow, he has played an active role in the Nanostructured Origami Project, which focuses on folding 3-D structures from 2-D nanopatterned precursors. His current research interests include developing mechanisms and processes that enable high volume industrial fabrication of complex nano-scaled devices.

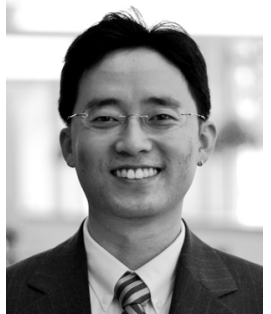

Se Baek Oh received the B.S. and M.S. degrees in mechanical engineering from the Korea Advanced Institute of Science and Technology, Yuseong, Daejeon, Korea, in 1999 and 2001, respectively, and the $\mathrm{Ph} . \mathrm{D}$. degree in mechanical engineering from the Massachusetts Institute of Technology (MIT), Cambridge, in 2009.

$\mathrm{He}$ is currently Post-Doctoral Associate of Mechanical Engineering with MIT. His current research interests include engineering of 3-D optical imaging systems based on volume holography and Wigner analysis with applications to multidimensional imaging and optical metrology.

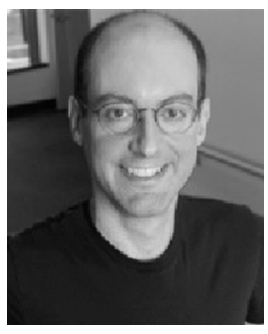

George Barbastathis received the B.Eng. degree in electrical and computer engineering from the National Technical University of Athens, Athens, Greece, in 1993, and the M.Sc. and Ph.D. degrees in electrical engineering from the California Institute of Technology, Pasadena, in 1994 and 1997, respectively.

After post-doctoral work with the University of Illinois at Urbana-Champaign, Champaign, he joined the Faculty at the Massachusetts Institute of Technology (MIT), Cambridge, in 1999, where he is currently a Professor of Mechanical Engineering. From 2006-2007, he was on leave from MIT as a Visiting Scholar with the Division of Engineering and Applied Sciences, Harvard University, Cambridge, MA. From 2008-2009, he was a Resident Faculty Member with the Singapore-MIT Alliance for Research and Technology Center, Singapore. His current research interests include 3-D microscopy, phase retrieval, dielectric metamaterial imaging, and micro-mechanically tunable optical systems.

Dr. Barbastathis is on the Technical Advisory Board of several start-up companies in the photonics and nanotechnology spaces, and has served as a Topical Editor for the Journal of the Optical Society A. 